\section{On the orientation of the pulsar space velocity vector relative to the spin axis}

A SIGNIFICANT parameter for pulsars for which proper motion data are available is the angle $\varphi$ between the space velocity vector and the spin axis. There are eleven such pulsars (ref. 15 and personal communication from one of the authors). The angle between the spin axis projected on the plane of the sky and the proper motion vector has a value close to $0^{\circ}$ for three of them, close to $90^{\circ}$ for six and between $0^{\circ}$ and $90^{\circ}$ for the remaining two. We present here a hypothesis which would explain how the different orientations of the space velocity vector relative to the spin axis arise. We distinguish between three modes of formation of pulsars and show that these correspond to the three different sets of values of the angle.

The supernova explosion of a rotating magnetised star would result in the ejection of double jets along the spin axis ${ }^{1}$, and only a part of the stored magnetic energy is sufficient to eject the supernova shell ${ }^{2}$. Since in real stars the field is usually not at all symmetrical between the two poles, one would expect the jet ejection to be an asymmetric process resulting in the neutron star getting a kick along the spin axis. Asymmetric radiation, due to the dipole being off centre, from the resulting pulsar could give it an additional kick along the spin axis ${ }^{3}$. In other words, pulsars formed from the supernova explosions of single stars should have space velocities along the spin axis; pulsars with $\varphi \approx 0^{\circ}(0531+21,0833-45$ and $2016+28)$ belong to this category.

For a supernova explosion to take place in a close binary system, the primary should have a mass $\gtrsim 15 M_{\odot}$ (ref. 4). The primary evolves quickly, transferring more than two thirds of its mass to the companion before it explodes as a supernova, leaving behind a relativistic star: a neutron star or a black hole. Since the more evolved component is also the lighter (at the time of the explosion), mass ejection will not disrupt the binary ${ }^{5}$.

Two other mechanisms are available in principle for disrupting the binary. (1) The ejected supernova shell could impart a critical impulse to the unexploded companion making it leave the system ${ }^{6}$. It has been shown ${ }^{7}$, however, that this mechanism is not likely to be effective. (2) A highly asymmetric supernova explosion could impart a sufficient kick to the exploding star to push it into a hyperbolic orbit. To disrupt the binary in this manner, the impulse should be imparted in the direction of the orbital motion ${ }^{8}$. It is extremely unlikely that a kick normal to the orbital plane-assuming of course that the spin axis is perpendicular to the orbital plane-would disrupt the system. Highest observed space velocity for single pulsars is around $400 \mathrm{~km} \mathrm{~s}^{-1}$ (ref. 9). Velocities of this order are definitely unable to disrupt a binary system especially if the kick is normal to the orbital plane. So the first supernova explosion leaving behind a relativistic star core is unlikely to disrupt the binary. However, to account for the existence of runaway stars, it is necessary to disrupt some of the binaries at this stage. It should therefore be conceded that some supernova explosions do not leave behind any core ${ }^{10}$.

If a neutron star were formed in a massive binary it might tilt the orbital plane and impart a velocity of a few dozens of kilometres per second to the centre of the mass of the binary. It could also make the orbit eccentric but this would eventually become circular due to tidal action forcing the $O B$ type main sequence star into synchronous rotation ${ }^{11}$. The binary would manifest itself as an X-ray source as long as mass being accreted by the compact star from the OB type star (now a blue supergiant) is large enough to give a measurable $\mathrm{X}$-ray flux ${ }^{12}$, but not too large to choke the source ${ }^{13}$. Large mass loss would ensue when the primary evolves beyond the blue supergiant stage and overflows the Roche lobe. The X-ray source would be extinguished and matter start flowing out from the outer Lagrangian point. Enhanced accretion by the neutron star and mass loss from the system would spin up the neutron $\operatorname{star}^{14}$.
The explosion of the resulting helium star is likely to disrupt the system because it is more massive than the companion neutron star. (If the companion is a black hole, disruption probability would be smaller because then both the members of the system will have comparable masses). The disruption of the orbit would release two neutron stars. The old, reactivated neutron star would move in the orbital plane with its orbital velocity. So it would have a space velocity normal to the spin axis $^{15}$. This is how pulsars with $\varphi \approx 90^{\circ}(0823+26,0834+06$, $1133+16,1237+25,1929+10$ and $2021+51$ ) were liberated from binary orbits which were disrupted by the supernova explosions of the companion stars. The exploding star would get a kick along the spin axis ${ }^{1}$ and so it will move with a velocity equal to the vector sum of the mutually perpendicular kick velocity and its orbital velocity. Pulsars with $0<\varphi<90^{\circ}(0329+54$ and $0950+08)$ are thus produced by the supernova explosions in and leading to the disruption of the binary systems.

I thank Dr Rajamohan and Mr T. P. Prabhu for useful discussion and Dr C. Shukre who very kindly updated the data of their paper for my use.

\section{R. K. KOCHHAR}

Indian Institute of Astrophysics, Bangalore-560034, India

Received 2 February; accepted 4 March 1977

1 Le Blanc, J. M. \& Wilson, J. R. Astrophys. J. 161, 541 (1970).

Kundt, W. Nature 261, 673 (1976)

Tademaru, E. \& Harrison, E. R. Nature 254, 676 (1975)

Van den Heuvel, E. P. J. Astrophysics and Gravitation. Proc. 16th Solvay Conf. p.119 (University of Brussels Press, 1974).

${ }^{5}$ Boersma, J. Bull. Astron. Inst. Netherlands 15, 291 (1961).

Sofia, S. Astrophys. J. 149, L59 (1967)

Sutantyo, W. Astron. Astrophys. 31, 339 (1974).

De Loore, C. \& van den Heuvel, E. P. J. Astron. Astrophys. 52, $315(1976)$

Manchester, R. N., Taylor, J. H. \& Van, Y. T. Astrophys. J. 189, L1 19 (1974)

Arnett, W. D. Astrophys. Space Sci., 5, 180 (1969).

Lea, S. M. \& Margon, B. Astrophys. Lett. 13, 33 (1973).

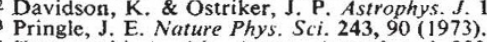

34 Pringle, J. E. Nature Phys. Sci. 243, 90 (1973).

is Morris, D., Radhakrishnan, V. \& Shukre, C. Nature 254, 676 (1975).

\section{How did barium titanate particulates stick together in the nebula?}

TANAKA et al. ${ }^{1}$ have suggested that there must be barium-rich micro-components in the Allende meteorite. We have confirmed this prediction by discovery of barium-rich particulates (see ref. 2). Figure 1 shows diffuse distribution of Ba-rich particles as well as their clusters; each of apparent particles seems to be composed of finer particulates probably of submicron size. Tanaka and Okumura ${ }^{2}$ showed that the Ba-rich spots are also high in content of titanium. Figure 2 shows the areal overlapping of the distribution of $\mathrm{Ba}$ with that of $\mathrm{Ti}$. Relatively sparse dots for $\mathrm{Ti}$ are due to shorter scanning time than for $\mathbf{B a}$.

The results of electron microprobe studies ${ }^{2}$ show the atomic ratio of $\mathrm{Ba}$ to $\mathrm{Ti}$ to be almost $1: 1$. Moreover, based on the data obtained by Tanaka and Okumura ${ }^{2}$, contents of $\mathrm{BaO}$ and $\mathrm{TiO}_{2}$ in most $\mathrm{Ba}$-Ti-rich portion turn out to be 59 and $34 \%$ respectively; the pure chemical formula $\mathrm{BaTiO}_{3}$ gives the theoretical values $66 \% \mathrm{BaO}$ and $34 \% \mathrm{TiO}_{2}$. Thus the purity of micro-component as barium titanate is amazingly high. The small difference between actual and theoretical contents may be due either to experimental errors or to the presence of small amounts of other titanates and probably silicates. Palme and Wlotzka ${ }^{3}$ separated a particle (about $20 \mu \mathrm{m}$ ) rich in refractory siderophile elements Os, W, Re, Ir, Mo, Ru and Pt from the Allende meteorite.

The $\mathrm{Ba}$ content of the Allende $\mathrm{BaTiO}_{3}$ is therefore up to $1.7 \times 10^{5}$ times the corresponding chondritic abundance. 